\title{
ADK divergence measure and testing exponentiality based on estimated ADK information
}

\author{
Morteza Khodabin \\ Department of Mathematics, College of basic sciences, Karaj Branch, Islamic Azad University, Alborz, Iran \\ Email: m-khodabin@kiau.ac.ir
}

Copyright (c)2014 Morteza Khodabin. This is an open access article distributed under the Creative Commons Attribution License, which permits unrestricted use, distribution, and reproduction in any medium, provided the original work is properly cited.

\begin{abstract}
In this paper, I define the two parameter ADK divergence measure, as a generalized of Re'nyi divergence measure. Also I introduce a goodness of fit tests for exponentiality based on the estimated ADK information. I use an estimator for ADK distance in manner of Correa estimate. Critical values of the test are obtained by Monte Carlo simulation. The power of the test is computed under gamma distribution.
\end{abstract}

Keywords: KADK entropy; Renyi entropy; Shannon entropy; Kullback - Leibler divergence measure; Renyi divergence measure; ADK divergence measure; Exponentiality test; Critical value

\section{Introduction}

The concept of Shannon's entropy [8] is the central role of information theory sometimes referred as measure of uncertainty. The entropy of a random variable is defined in terms of its probability distribution and can be shown to be a good measure of randomness or uncertainty. The concept of entropy and entropy-like quantities have proved to be instrumental in many fields of science. A large number of entropy-like quantities have appeared in the scientific literature. All these new quantities share some but not all properties with the Shannon entropy. The most important examples are the Renyi entropy and the Tsallis entropy. There are so many other definitions of entropy-like quantities that Arndt [7] was able to write a whole book entitled "Information Measures". The Renyi entropy was introduced by Renyi $[4,5]$ and soon after it found application in graph theory. The original reason for Renyi to introduce his new entropy is said to be that he planned to use it in an information theoretic proof of the central Limit Theorem. Aczel and Daroczy [12], Kapur [13], have introduced a general form of entropy,which Khodabin $[14,15]$ called it ADK entropy.

Furthermore, testing exponentiality is a long standing problem in statistics. The exponential distribution is needed and used at least as often as the time-homogeneous Poisson process. Applications of the exponential distribution in physics, and more so in survival analysis and reliability theory, are numerous and diverse [19].

Many authors such as Lillifors, H. W. [17], Van-Soset, J. [21], Finkelsein, J. and Schafer, R.E. [11] have introduced different test statistics for exponentiality hypothesis testing. Ebrahimi et Al. [10] make use of the maximum entropy characterization by considering the difference between a nonparametric estimator of entropy and the maximum likelihood estimator of entropy under exponentiality. Other omnibus tests for exponentiality have been developed by Henze [11], Baringhaus and Henze [6] who introduced tests of fit for exponentiality based on a characterization via the mean residual life function. 
Most recently, Zamanzadeh, E. and Arghami, N. R. [23] discussed normality and exponentiality tests based on new entropy estimators. Yousefzadeh, F. and Arghami, N.R. [22] have been presented testing exponentiality based on type II censored data and new cdf estimator. Alizadeh Noughabi H. and et al. [2,3] compare the different entropy estimators and power of exponentiality tests based on entropy estimators. Abbasnejad, M. and Shakuri, M. [1] propose a goodness of fit test for exponentiality based on estimated Renyi information.

Here, I extend their work and suggest ADK divergence measure and testing exponentiality based on estimated ADK information as special case of Renyi information.

This paper is organized as follows:

In Section 2, the ADK entropy and joint ADK entropy are presented. In Section 3, the ADK divergence measure is defined. In section 4, the Correa estimation of ADK divergence measure is introduced and is used as test statistics. In section 5 , the critical and power values of test are evaluated at 0.05 significant level for some different values of $\alpha, \beta$ and $n$. Finally Section 6 briefly considers the conclusions.

\section{ADK entropy}

In information theory, the ADK entropy, as a generalization of Renyi and Shannon entropy, is one of a family of functional for quantifying the diversity, uncertainty or randomness of a system. It is named ADK because Aczel, Daroczy and Kapur initial works. For a review of earlier contributions, the interested reader is referred to [14,15].

Suppose that:

1. All integrals and sums exist.

2. $\alpha, \beta>0, \alpha \neq 1$.

3. $\log$ is to the base 2 and entropy is expressed in bits.

Let $\left(S, \beta_{S}, P\right)_{p} \in \Delta_{n}$ be an statistical space, where $S=\left\{x_{1}, x_{2}, \ldots, x_{n}\right\}, \Delta_{n}=\left\{P=\left(p_{1}, p_{2}, \ldots, p_{n}\right), p_{i} \geq\right.$ $\left.0, \sum_{i=1}^{n} p_{i}=1\right\}$ and $\beta_{S}$ is the $\sigma$-field of all the subsets of $S$. [14] ADK entropy of any probability distribution $P=\left(p_{1}, p_{2}, \ldots, p_{n}\right)$ of a random variable is defined as

$H_{\alpha, \beta}(X) \equiv H_{\alpha, \beta}(P)=\frac{1}{1-\alpha} \log \frac{\sum_{i=1}^{n} p_{i}^{\alpha+\beta-1}}{\sum_{i=1}^{n} p_{i}^{\beta}}$.

Two particular cases of this entropy can be written in the forms

$$
H_{\alpha, 1}(X) \equiv H_{\alpha}(X)=\frac{1}{1-\alpha} \log \sum_{i=1}^{n} p_{i}^{\alpha},
$$

which is the Re'nyi entropy of order $\alpha$, and

$$
\lim _{\alpha \rightarrow 1} H_{\alpha, 1}(X) \equiv H_{1}(X)=-\sum_{i=1}^{n} p_{i} \log p_{i}
$$

which is the Shannon entropy. (The joint ADK entropy [14]): If $X_{1}, X_{2}, \ldots, X_{n}$ are distributed according to $p\left(x_{1}, x_{2}, \ldots, x_{n}\right)$ then

$H_{\alpha, \beta}\left(X_{1}, X_{2}, \ldots, X_{n}\right)=\frac{1}{1-\alpha} \log \frac{\sum_{x_{1}, x_{2}, \ldots, x_{n}} p\left(x_{1}, x_{2}, \ldots, x_{n}\right)^{\alpha+\beta-1}}{\sum_{x_{1}, x_{2}, \ldots, x_{n}} p\left(x_{1}, x_{2}, \ldots, x_{n}\right)^{\beta-1}}$

Let $X$ be a random variable having an absolutely continuous distribution with density function $f(x)$.

[15] The ADK entropy of order $\alpha$ and $\beta$ for continuous random variable is defined as

$H_{\alpha, \beta}(X)=\frac{1}{1-\alpha} \log \frac{\int_{-\infty}^{+\infty} f^{\alpha+\beta-1}(x) d x}{\int_{-\infty}^{+\infty} f^{\beta}(x) d x}$.

Two particular cases of this family can be given by

$H_{\alpha, 1}(X)=\frac{1}{1-\alpha} \log \int_{-\infty}^{+\infty} f^{\alpha}(x) d x$, 
which is the Renyi entropy of order $\alpha$, and

$H(X)=\lim _{\alpha \rightarrow 1} H_{\alpha, 1}(X)=-\int_{-\infty}^{+\infty} f(x) \log f(x) d x$,

is the Shannon entropy. [15] If $\left(X_{1}, \ldots, X_{n}\right)$ be a random vector with density function $f\left(x_{1}, \ldots, x_{n}\right)$ then

$H_{\alpha, \beta}\left(X_{1}, \ldots, X_{n}\right)=\frac{1}{1-\alpha} \log \frac{\int_{R^{n}} f^{\alpha+\beta-1}\left(x_{1}, \ldots, x_{n}\right) d x_{1} \ldots d x_{n}}{\int_{R^{n}} f^{\beta}\left(x_{1}, \ldots, x_{n}\right) d x_{1} \ldots d x_{n}}$

\section{ADK divergence measure}

Unless otherwise specified, it is understood that $0 \log 0=0 \log \frac{0}{0}=0$, If $q_{i}=0$ for some $i$, the corresponding $p_{i}$ is also zero. Kullback-Leibler divergence (K-L divergence)[16] is a non-symmetric measure of the difference between two probability distributions $\mathrm{P}$ and $\mathrm{Q}$. For probability distributions $P$ and $Q$ (of a discrete random variable) their K-L divergence is defined to be

$$
D_{\mathrm{KL}}(P \| Q)=\sum_{i=1}^{n} p(i) \log \frac{P(i)}{q(i)}
$$

In words, it is the average of the logarithmic difference between the probabilities $P$ and $Q$, where the average is taken using the probabilities $P$.

For continuous random variable, K-L divergence is defined to be the integral

$$
D_{\mathrm{KL}}(f \| g)=\int_{-\infty}^{\infty} f(x) \log \frac{f(x)}{g(x)} d x,
$$

where $f(x)$ and $g(x)$ denote the densities functions. Renyi divergence measure of a discrete random variable (of order $\alpha$ ) is defined [4] as

$$
D_{\alpha}(p \| q)=\frac{1}{1-\alpha} \log \sum_{i=1}^{n} \frac{p_{i}^{\alpha}}{q_{i}^{\alpha-1}} .
$$

For continuous random variable, Renyi divergence is defined to be the integral

$$
D_{\alpha}(f \| g)=\frac{1}{1-\alpha} \log \int_{-\infty}^{+\infty} \frac{f^{\alpha}(x)}{g^{\alpha-1}(x)} d x .
$$

Now, in this correspondence, I introduce a new divergence measure that overcomes the previous definitions. (ADK divergence measure). The regular relations for ADK divergence measure of two type distributions are given by

$D_{\alpha, \beta}(p \| q)=\frac{1}{1-\alpha} \log \frac{\sum_{i=1}^{n} \frac{p_{i}^{\alpha+\beta-1}}{q_{i}^{\alpha+\beta-2}}}{\sum_{i=1}^{n} \frac{p_{i}^{\beta}}{q_{i}^{\beta-1}}}$,

and

$D_{\alpha, \beta}(f \| g)=\frac{1}{1-\alpha} \log \frac{\int_{-\infty}^{+\infty} \frac{f^{\alpha+\beta-1}(x)}{g^{\alpha+\beta-2}(x)} d x}{\int_{-\infty}^{+\infty} \frac{f^{\beta}(x)}{g^{\beta-1}(x)} d x}$,

respectively. Its explicit that, if we put $\beta=1$ we get Renyi divergence measure and if moreover $\alpha \rightarrow 1$ we have KL-divergence measure. 


\section{Test of exponentiality}

The exponential distribution occurs naturally when describing the lengths of the inter arrival times in a homogeneous Poisson process. Exponential variables can also be used to model situations where certain events occur with a constant probability per unit length, such as the distance between mutations on a DNA strand, or between road kills on a given road. In queuing theory, the service times of agents in a system (e.g. how long it takes for a bank teller etc. to serve a customer) are often modeled as exponentially distributed variables. Reliability theory and reliability engineering also make extensive use of the exponential distribution. Because of the memoryless property of this distribution, it is well-suited to model the constant hazard rate portion of the bathtub curve used in reliability theory.

Let $X$ be a nonnegative random variable with distribution function $F$. Consider the following hypothesis

$H_{0}: f(x)=f_{0}(x, \theta) \quad$ versus $\quad H_{1}: f(x) \neq f_{0}(x, \theta)$,

where,

$$
f_{0}(x, \theta)=\theta e^{-\theta x}, \quad x>0, \quad \theta>0 .
$$

The ADK distance between $f(x)$ and $f_{0}(x, \theta)$ is given by

$$
\begin{array}{r}
D_{\alpha, \beta}\left(f \| f_{0}\right)=-\log \theta+\frac{1}{\alpha-1} \log \int_{0}^{1}\left[\frac{d}{d p} F^{-1}(p)\right]^{2-\alpha-\beta} \exp \left\{\theta(\alpha+\beta-2) F^{-1}(p)\right\} d p \\
-\frac{1}{\alpha-1} \log \int_{0}^{1}\left[\frac{d}{d p} F^{-1}(p)\right]^{1-\beta} \exp \left\{\theta(\beta-1) F^{-1}(p)\right\} d p
\end{array}
$$

Proof: $\operatorname{From}(3.2)$ we have

$$
D_{\alpha, \beta}\left(f \| f_{0}\right)=\frac{1}{\alpha-1} \log \int_{0}^{\infty} \frac{f^{\alpha+\beta-1}(x)}{\left(\theta e^{-\theta x}\right)^{\alpha+\beta-2}} d x-\frac{1}{\alpha-1} \log \int_{0}^{\infty} \frac{f^{\beta}(x)}{\left(\theta e^{-\theta x}\right)^{\beta-1}} d x .
$$

By putting $F(x)=p$ and somedeal simplification we get (4.2).

Suppose that $X_{1}, X_{2}, \ldots, X_{n}$, be a random sample of size $n$ from a continuous distribution function $F(x)$. Let us set $X_{(1)}, X_{(2)}, \ldots, X_{(n)}$ for the order statistics that is defined by sorting the values $X_{1}, X_{2}, \ldots, X_{n}$ in increasing order. The Correa estimation of $D_{\alpha, \beta}\left(f \| f_{0}\right)$ consist of

$$
\begin{aligned}
D_{c}(\alpha, \beta)=-\log \bar{X}+ & \frac{1}{\alpha-1} \log \left[\frac{1}{n} \sum_{i=1}^{n}\left\{w_{i} \exp \left(X_{(i)} \bar{X}\right)\right\}^{\alpha+\beta-2}\right] \\
& -\frac{1}{\alpha-1} \log \left[\frac{1}{n} \sum_{i=1}^{n}\left\{w_{i} \exp \left(X_{(i)} \bar{X}\right)\right\}^{\beta-1}\right],
\end{aligned}
$$

where,

$$
\bar{X}_{(i)}=\frac{\sum_{j=i-m}^{i+m} X_{(j)}}{2 m+1}
$$

and

$$
w_{j}=\frac{\sum_{j=i-m}^{i+m}\left(X_{(j)}-\bar{X}_{(i)}\right)(j-i)}{n \sum_{j=i-m}^{i+m}\left(X_{(j)}-\bar{X}_{(i)}\right)^{2}} .
$$

Also

$$
X_{(j)}=X_{(1)} \text { for } j<1 \text { and } X_{(j)}=X_{(n)} \text { for } j>n .
$$

Proof: By using Correa [1] estimation of entropy for (4.2)we get (4.3).

$H_{0}$ is rejected at the level $\epsilon$ for for sufficiently large value of $D_{c}(\alpha, \beta)$, i.e. $H_{0}$ is rejected if

$$
D_{c}(\alpha, \beta) \geq d(\epsilon)
$$

where the critical value $d(\epsilon)$ is obtained from $\epsilon$ th quartile under $H_{0}$. 


\section{Critical values and powers of the test}

The test statistics derived in the previous sections is too complicated to allow deriving it exact distribution under the null hypothesis analytically. The critical values of the test are obtained by a simulation using 10,000 samples of sizes 10,20,30,50,100. The results are tabulated for $\alpha=0.05$ in tables $1,3,5,7$ and 9 .

The powers of the test for gamma alternative are also tabulated in tables $2,4,6,8$ and 10 .

\begin{tabular}{|c|c|c|c|c|c|}
\hline$\overline{\alpha \backslash \beta}$ & 0.2 & 0.5 & 0.9 & 1 & 1.2 \\
\hline 0.2 & 0.803591 & 0.8302 & 0.903887 & 0.949262 & 1.107318 \\
\hline 0.5 & 0.807452 & 0.840762 & 0.961392 & 1.032446 & 1.247746 \\
\hline 0.9 & 0.832644 & 0.883796 & 1.096901 & 1.240068 & 1.684263 \\
\hline 0.99 & 0.831725 & 0.898237 & 1.163079 & 1.289637 & 1.786265 \\
\hline 0.999 & 0.83778 & 0.894969 & 1.162233 & 1.310809 & 1.834924 \\
\hline 1.1 & 0.84943 & 0.916316 & 1.220923 & 1.416044 & 2.045871 \\
\hline 1.3 & 0.858195 & 0.958164 & 1.43453 & 1.748441 & 2.493469 \\
\hline 1.5 & 0.8826 & 1.030367 & 1.786868 & 2.121369 & 3.107706 \\
\hline
\end{tabular}

Table 2: Power of the test statistics at some different values $\alpha$ and $\beta$ for $\mathrm{n}=10$.

\begin{tabular}{cccccc}
\hline$\alpha \backslash \beta$ & 0.2 & 0.5 & 0.9 & 1 & 1.2 \\
\hline 0.2 & 0.9868 & 0.9734 & 0.793 & 0.5818 & 0.1136 \\
0.5 & 0.9864 & 0.965 & 0.5187 & 0.2757 & 0.0205 \\
0.9 & 0.9694 & 0.8734 & 0.134 & 0.0225 & 0 \\
0.99 & 0.9748 & 0.82 & 0.0612 & 0.0133 & 0 \\
0.999 & 0.9665 & 0.8327 & 0.0566 & 0.0089 & 0 \\
1.1 & 0.9484 & 0.7445 & 0.0303 & 0.0018 & 0 \\
1.3 & 0.9398 & 0.5433 & 0.0012 & 0 & 0 \\
1.5 & 0.8816 & 0.2787 & 0 & 0 & 0 \\
\hline
\end{tabular}

Table 3: Critical values of the test statistics at some different values $\alpha$ and $\beta$ for $\mathrm{n}=20$.

\begin{tabular}{cccccc}
\hline$\alpha \backslash \beta$ & 0.2 & 0.5 & 0.9 & 1 & 1.2 \\
\hline 0.2 & 0.617612 & 0.645722 & 0.703835 & 0.725462 & 0.803851 \\
0.5 & 0.630272 & 0.6633 & 0.73444 & 0.774155 & 0.874317 \\
0.9 & 0.647323 & 0.687574 & 0.801435 & 0.864544 & 1.070318 \\
0.99 & 0.649255 & 0.698442 & 0.829386 & 0.896908 & 1.160357 \\
0.999 & 0.651827 & 0.701768 & 0.83493 & 0.905643 & 1.158742 \\
1.1 & 0.657161 & 0.712093 & 0.867486 & 0.96143 & 1.266109 \\
1.2 & 0.672906 & 0.735791 & 0.966313 & 1.08155 & 1.580651 \\
1.5 & 0.691403 & 0.769994 & 1.134302 & 1.368023 & 2.044367 \\
\hline
\end{tabular}

Table 4: Power of the test statistics at some different values $\alpha$ and $\beta$ for $\mathrm{n}=20$.

\begin{tabular}{cccccc}
\hline$\alpha \backslash \beta$ & 0.2 & 0.5 & 0.9 & 1 & 1.2 \\
\hline 0.2 & 1 & 1 & 1 & 1 & 1 \\
0.5 & 1 & 1 & 1 & 1 & 0.9802 \\
0.9 & 1 & 1 & 1 & 0.9893 & 0.1369 \\
0.99 & 1 & 1 & 0.999 & 0.9434 & 0.0298 \\
0.999 & 1 & 1 & 0.9989 & 0.9162 & 0.0323 \\
1.1 & 1 & 1 & 0.9869 & 0.626 & 0.0071 \\
1.2 & 1 & 1 & 0.5986 & 0.1077 & 0.0002 \\
1.5 & 1 & 1 & 0.0427 & 0.0024 & 0 \\
\hline
\end{tabular}




\begin{tabular}{cccccc}
\multicolumn{6}{c}{ Table 5: Critical values of the test statistics at some different values $\alpha$ and $\beta$ for $\mathrm{n}=30$. } \\
\hline \multicolumn{1}{c}{$\backslash$} & 0.2 & 0.5 & 0.9 & 1 & 1.2 \\
\hline 0.2 & 0.540659 & 0.565438 & 0.622921 & 0.642392 & 0.705734 \\
0.5 & 0.554531 & 0.5847 & 0.649142 & 0.678615 & 0.756976 \\
0.9 & 0.568029 & 0.606536 & 0.708125 & 0.754242 & 0.911599 \\
0.99 & 0.574473 & 0.618336 & 0.723519 & 0.772528 & 0.949633 \\
0.999 & 0.572643 & 0.617668 & 0.730179 & 0.779016 & 0.955915 \\
1.1 & 0.57859 & 0.625259 & 0.75059 & 0.810281 & 1.029024 \\
1.2 & 0.59262 & 0.648213 & 0.81841 & 0.903993 & 1.230523 \\
1.5 & 0.611542 & 0.677173 & 0.93446 & 1.085828 & 1.605678 \\
\hline
\end{tabular}

Table 6: Power of the test statistics at some different values $\alpha$ and $\beta$ for $\mathrm{n}=30$.

\begin{tabular}{cccccc}
\hline$\alpha \backslash \beta$ & 0.2 & 0.5 & 0.9 & 1 & 1.2 \\
\hline 0.2 & 1 & 1 & 1 & 1 & 1 \\
0.5 & 1 & 1 & 1 & 1 & 1 \\
0.9 & 1 & 1 & 1 & 1 & 0.9357 \\
0.99 & 1 & 1 & 1 & 1 & 0.7583 \\
0.999 & 1 & 1 & 1 & 1 & 0.7222 \\
1.1 & 1 & 1 & 1 & 1 & 0.2273 \\
1.2 & 1 & 1 & 1 & 0.9603 & 0.0066 \\
1.5 & 1 & 1 & 0.8445 & 0.0648 & 0 \\
\hline
\end{tabular}

Table 7: Critical values of the test statistics at some different values $\alpha$ and $\beta$ for $\mathrm{n}=50$.

\begin{tabular}{cccccc}
\hline$\alpha \backslash \beta$ & 0.2 & 0.5 & 0.9 & 1 & 1.2 \\
\hline 0.2 & 0.467814 & 0.495983 & 0.545806 & 0.564894 & 0.616745 \\
0.5 & 0.478874 & 0.508411 & 0.570922 & 0.594333 & 0.665378 \\
0.9 & 0.4961 & 0.533694 & 0.623415 & 0.657909 & 0.76766 \\
0.99 & 0.503115 & 0.539602 & 0.635884 & 0.67711 & 0.801938 \\
0.999 & 0.499412 & 0.541323 & 0.635479 & 0.682642 & 0.808344 \\
1.1 & 0.507584 & 0.549387 & 0.659474 & 0.706458 & 0.853206 \\
1.2 & 0.520215 & 0.569823 & 0.709839 & 0.777926 & 0.98735 \\
1.5 & 0.536557 & 0.596974 & 0.790137 & 0.883672 & 1.218955 \\
\hline
\end{tabular}

Table 8: Power of the test statistics at some different values $\alpha$ and $\beta$ for $\mathrm{n}=50$.

\begin{tabular}{cccccc}
\hline$\alpha \backslash \beta$ & 0.2 & 0.5 & 0.9 & 1 & 1.2 \\
\hline 0.2 & 1 & 1 & 1 & 1 & 1 \\
0.5 & 1 & 1 & 1 & 1 & 1 \\
0.9 & 1 & 1 & 1 & 1 & 1 \\
0.99 & 1 & 1 & 1 & 1 & 1 \\
0.999 & 1 & 1 & 1 & 1 & 1 \\
1.1 & 1 & 1 & 1 & 1 & 1 \\
1.2 & 1 & 1 & 1 & 1 & 0.514 \\
1.5 & 1 & 1 & 1 & 0.9977 & 0.0025 \\
\hline
\end{tabular}

Table 9: Critical values of the test statistics at some different values $\alpha$ and $\beta$ for $\mathrm{n}=100$.

\begin{tabular}{cccccc}
\hline$\alpha \backslash \beta$ & 0.2 & 0.5 & 0.9 & 1 & 1.2 \\
\hline 0.2 & 0.395754 & 0.421483 & 0.472232 & 0.491045 & 0.534749 \\
0.5 & 0.406919 & 0.437238 & 0.496413 & 0.518442 & 0.577834 \\
0.9 & 0.424957 & 0.460256 & 0.540542 & 0.569828 & 0.662374 \\
0.99 & 0.429322 & 0.467234 & 0.552902 & 0.589397 & 0.68971 \\
0.999 & 0.428159 & 0.467135 & 0.554794 & 0.591341 & 0.691894 \\
1.1 & 0.432718 & 0.476208 & 0.57253 & 0.609951 & 0.725397 \\
1.2 & 0.446727 & 0.493305 & 0.613218 & 0.666919 & 0.814188 \\
1.5 & 0.460665 & 0.518232 & 0.676025 & 0.74367 & 0.934898 \\
\hline
\end{tabular}




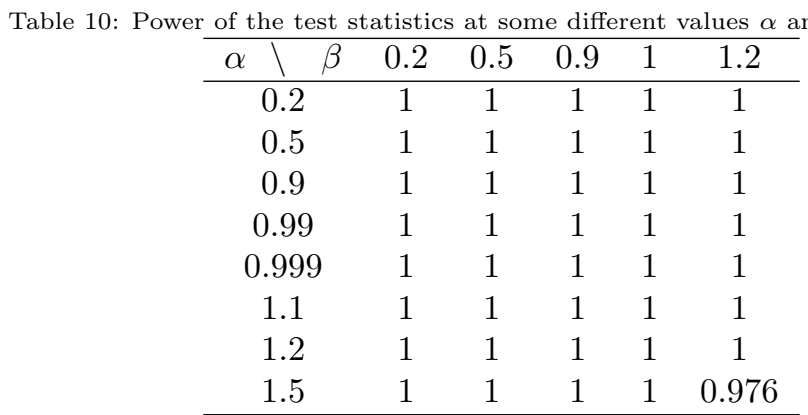

\section{Conclusion}

I introduced the ADK divergence measure as a generalization of Renyi distance and used it as a test statistics for goodness of fit test for exponentioality. In general we can say, since this test statistics has two paprameters in comparison with estimated Renyi information, we can select a tests with high powers. Furthermore, look at the below table which summarize above tables.

\begin{tabular}{|c|c|c|c|c|c|}
\hline$\alpha \backslash \beta$ & 0.2 & 0.5 & 0.9 & 1 & 1.2 \\
\hline 0.2 & $(+,+,+,+,+)$ & $(+,+,+,+,+)$ & $(-,+,+,+,+)$ & $(-,+,+,+,+)$ & $(-,+,+,+,+)$ \\
\hline 0.5 & $(+,+,+,+,+)$ & $(+,+,+,+,+)$ & $(-,+,+,+,+)$ & $(-,+,+,+,+)$ & $(-,+,+,+,+)$ \\
\hline 0.9 & $(+,+,+,+,+)$ & $(-,+,+,+,+)$ & $(-,+,+,+,+)$ & $(-,+,+,+,+)$ & $(-,-,-,+,+)$ \\
\hline 0.99 & $(+,+,+,+,+)$ & $(-,+,+,+,+)$ & $(-,+,+,+,+)$ & $(-,+,+,+,+)$ & $(-,-,-,+,+)$ \\
\hline 0.999 & $(+,+,+,+,+)$ & $(-,+,+,+,+)$ & $(-,+,+,+,+)$ & $(-,+,+,+,+)$ & $(-,-,-,+,+)$ \\
\hline 1.1 & $(+,+,+,+,+)$ & $(-,+,+,+,+)$ & $(-,+,+,+,+)$ & $(-,-,+,+,+)$ & $(-,-,-,+,+)$ \\
\hline 1.2 & $(+,+,+,+,+)$ & $(-,+,+,+,+)$ & $(-,-,+,+,+)$ & $(-,-,+,+,+)$ & $,-,-,+)$ \\
\hline 1.5 & $(-,+,+,+,+)$ & $(-,+,+,+,+)$ & $(-,-,-,+,+)$ & $(-,-,-,+,+)$ & $(-,-,-,-,+)$ \\
\hline
\end{tabular}

In the five-ply $(., ., ., .,$.$) , the +$ sign shows that the test has a high power (power $>0.9)$ and is accessible. The first component is used for $n=10$, the second is used for $n=20$ and so on. From these signs we can conclude that :

For a large sample size $(n \geq 50)$, the test has a high power for all values of $\alpha$ and $\beta$, but for a small sample size ( Here $\mathrm{n}=10,20,30$ ), the test has a high power only for some values of $\alpha$ and $\beta$.algorithm in this paper for calculating stresses around tunnels.

\section{References}

[1] Abbasnejad, M. and Shakuri, M. (2008), A goodness of fit test for exponentiality based ok estimated Renyi information, Journal of Statistical Sciences, Vol. 2, No. 2, 201-211.

[2] Alizadeh Noughabi H. , Arghami, N. R. and Alizadeh Noughabi R. (2008), Comparison of different entropy estimators and power of exponentiality tests based on entropy estimators, Journal of Statistical Sciences, Vol. 2, No. 2, $213-227$.

[3] Alizadeh Noughabi H. , Arghami, N. R. (2011), Monte Carlo comparison of five exponentiality tests using different entropy estimates. Journal of Statistical Computation and Simulation Vol. 81, No. 11, November $2011,15791592$.

[4] A. Renyi, 1961, On measures of entropy and information, in: Proceedings of the Fourth Berkeley Symposium on Mathematics, Statistics and Probability, vol. 1, University California Press, Berkeley, 547-561.

[5] A. Renyi, 1965, On the foundation of information theory, Rev. Inst. Int. Stat. 33 1-14.

[6] Baringhaus, L. and Henze, N. (2000). Tests of fit for exponentiality based on a characterization via the mean residual life function, Statistical Papers 41, 225-236.

[7] C. Arndt, 2001, Information Measures, Springer, Berlin.

[8] C.E. Shannon, 1984, A mathematical theory of communication, Bell Syst. Tech. J 27 379-423. 623-656.

[9] Ebrahimi, N., Soofi, E.S. and Habibullah, M. (1992). Testing Exponentiality Based on Kullback-Leibler Information, Journal of the Royal Statistical Society B 54, 739-748. 
[10] Finkelsein, J. and Schafer, R.E. (1971), Imported goodness of fit tests, Biometrika, 58, 641-645.

[11] Henze, N. (1993). A New Flexible Class of Omnibus Tests for Exponentiality, Communications in Statistics - Theory and Methods 22, 115-133.

[12] J. Aczel and Z. Daroczy, 1963, Characterisierung der entropien positiver ordnung und der Shannons chen entropie. Act.Math.Acad.Sci.Hunger 14, 95-121.

[13] J.N. Kapur, 1967, Generalized entropy of order and type . The Math. Seminar 4 78-82.

[14] Khodabin, M. , (2011), Some properties of ADK entropy and ADK entropy rate, Procedia Computer Science 3, 11701177.

[15] Khodabin, M. , (2010), ADK entropy and ADK entropy rate in irreducible aperiodic Markov chain and Gaussian processes, JIRSS, accepted manuscript, and it will appear in Vol.9, No. 2.

[16] Kullback, S. (1959), Information Theory and Statistics. New York: John Wiley.

[17] Lillifors, H. W. (1969), On the Kolmogorov test for the exponential distribution with mean unknown, Journal of American Statistical Association,64,387-389.

[18] Park, S. (2005), Testing exponentiality based on the Kullback- Leibler information with the type II censored data. IEEE Trans. on Rel., 54, 22-26.

[19] Pham, H. (Ed.), 2003. Handbook of Reliability Engineering. Springer, London.

[20] Van-Soset, J. (1969), Some goodness of fit tests for exponential distribution, Statistica Neerlandica, 23, 41-51.

[21] Vasicek, O. (1976), A test for normality based on sample entropy, Journal of the Royal Statistical Society, 38, 54-59.

[22] Yousefzadeh, F. and Arghami, N.R. (2008), Testing exponentiality based on type II censored data and new cdf estimator, Communications in Statistics, Simulation and Computation, 37, 1479-1499.

[23] Zamanzadeh, E. and Arghami, N. R. (2008), Normality and exponentiality tests based on new entropy estimators, Journal of Statistical Sciences, Vol. 2, No. 2, 179-200. 